[0212-7199 (2008) 25: 7; pp 349-352] ANALES DE MEDICINA INTERNA Copyright (C) 2008 ARAN EDICIONES, S.L.

AN. MED. INTERNA (Madrid) Vol. 25, N. ${ }^{\circ} 7$, pp. 349-352, 2008

\title{
Disfunción de cuerdas vocales secundaria a reflujo gastroesofágico
}

\author{
P. YOUNG, B. C. FINN, M. L. FOX, N. EMERY, J. E. BRUETMAN \\ Servicio de Clínica Médica. Hospital Británico. Buenos Aires (Argentina)
} GASTROESOPHAGEAL REFLUX AS A CAUSE OF VOCAL CORD
DYSFUNCTION

\begin{abstract}
RESUMEN
La disfunción de cuerdas vocales se caracteriza por la aducción paradójica de las mismas durante la inspiración, y ocurre con mayor frecuencia en mujeres jóvenes. Es un síndrome caracterizado por crisis habitualmente autolimitadas de tos, sibilancias, disnea y estridor inspiratorio, presentados en forma brusca. Su incidencia e historia natural son desconocidas. Puede coexistir o asemejar a un asma refractario. Originalmente los trastornos psicológicos eran la única causa conocida, aunque luego se describieron múltiples factores desencadenantes orgánicos como el reflujo gastroesofágico, ejercicio, sinusitis, distonía, tiroidectomía, irritantes respiratorios e infecciones de vías aéreas superiores. Para el diagnóstico se requiere una alta sospecha, y la realización de una laringoscopia directa objetivando la aducción de las cuerdas vocales durante la inspiración en el paciente sintomático. La terapia fonoudiológica y la psicoterapia han sido extensamente utilizadas, sin una evaluación prospectiva. Uno de los ejes centrales del manejo, es el tratamiento de la causa subyacente si esta se identifica. Reportamos dos casos de disfunción de las cuerdas vocales secundaria a reflujo gastroesofágico que evolucionaron en forma favorable con tratamiento específico.
\end{abstract}

PALABRAS CLAVE: Cuerdas vocales. Reflujo gastroesofágico. Diagnóstico. Tratamiento.

\begin{abstract}
S
Vocal cord dysfunction (VCD), is characterized by a paradoxical adduction of the vocal cords during inspiration, and occurs predominantly in young women. Common symptoms are cough, wheezing, episodic dyspnea, and inspiratory stridor. The true incidence and course of the disease are unknown, and it is usually self limited. It can coexist with, or mimic refractory asthma. Psychological disorders were thought to be the principal cause, subsequently multiple organic diseases have also been reported, like gastroesophageal reflux disease (GERD). Diagnosis is made by clinical suspicion and direct observation. The Gold standard for diagnosis is laryngoscopy with visualization of the paradoxical motion of the vocal cords when the patient is symptomatic. Speech therapy and psychotherapy have been used extensively without any prospective study. We report two cases of VCD associated with GERD, both with excellent respond to treatment.
\end{abstract}

KEY WORDS: Vocal cord. Gastroesophageal reflux. Diagnosis. Treatment.

Young P, Finn BC, Fox ML, Emery N, Bruetman JE. Disfunción de cuerdas vocales secundaria a reflujo gastroesofágico. An Med Interna (Madrid) 2008; 25: 349-352.

\section{INTRODUCCIÓN}

La disfunción de cuerdas vocales (DCV) se caracteriza por la aducción de las mismas durante la inspiración, lo que resulta en una limitación al flujo aéreo a nivel de la laringe (1). La primera descripción se atribuye a Dunglison (2), quien en 1842 describió un desorden de los músculos laríngeos en mujeres con histeria, denominándolo "croup histérico". Si bien la DCV, es la expresión que mejor explica este síndrome, existen otras denominaciones a saber: estridor de Munchausen, pseudoasma, obstrucción de la vía aérea superior (VAS) no orgánica o funcional, asma facticio, croup espasmódico, sibilancias laríngeas emocionales, obstrucción de VAS psicógena, disquinesia laríngea episódica, laringoespasmo paroxístico episódico, estridor laríngeo funcional, laringoespasmo inducido por ejercicio y síndrome de laringe irritable $(1,3)$. La verdadera incidencia y el curso clínico de la enfermedad son desconocidos. Los síntomas más comunes son sibilancias, tos, disnea episódica, estridor inspiratorio, disfonía, y dolor torácico (3). Históricamente se atribuyo esta enfermedad a distintos desordenes psicológicos, en los últimos años se han encontrado múltiples causas orgánicas entre las que se encuentra el reflujo gastroesofágico (RGE). Se han descrito hasta la fecha aproximadamente 1.500 casos $(1,3)$. Se presentan dos pacien-

Trabajo aceptado: 20 de marzo de 2008 
tes con DCV secundario a RGE, internados en el Hospital Británico entre los años 2006-2007 y una revisión de la literatura.

\section{CASOS APORTADOS}

Caso $n^{\circ}$ 1: paciente masculino de 72 años, con antecedentes de tabaquismo severo, enfermedad pulmonar obstructiva crónica, con enfisema centrolobulillar, cardiopatía isquémica y linfoma no Hodgkin folicular de bajo grado estadio IV A, que no requirió tratamiento. Presentó 3 episodios de disnea bruscos, que comenzaron con tos asociada a estridor laríngeo, disfonía y sibilancias altas que requirieron internación en unidad de terapia intensiva (UTI) en 2 oportunidades. Los tres episodios fueron autolimitados de 30 minutos a una hora de duración. Nunca presentó cianosis ni desaturación. Durante el segundo episodio se realizó una fibro-laringoscopia donde se evidenció las cuerdas vocales edematizadas, cierre glótico en inspiración con una pequeña luz en glotis posterior y mejoría progresiva luego de terminar el estudio. Sin signos de traqueomalacia. Se realizó en el momento agudo un estudio funcional respiratorio (EFR), que mostró el clásico cajón en la curva inspiratoria. Una seriada esófago-gastroduodenal (SEGD) evidenció RGE moderado. Cabe señalar que el paciente no tenía los síntomas clásicos de RGE. Se descartó compromiso laríngeo o traqueal por linfoma, enfermedad tromboembólica, enfermedad pulmonar o cardiaca. La analítica fue normal. Después del primer episodio inició tratamiento con corticoides, beta 2 inhalados de larga duración y tiotropio $18 \mu \mathrm{g} /$ día, sumado al tratamiento fonoudiológico con reeducación de la voz. No se realizó apoyo psicológico. Luego de segundo episodio con el diagnóstico de RGE inició omeprazol $40 \mathrm{mg}$ /día y domperidona $10 \mathrm{mg}$ cada 12 horas, el paciente discontinuó dicho tratamiento presentando el tercer episodio. Luego de éste último y con la medicación mencionada lleva 10 meses asintomático.

Caso $n^{\circ}$ 2: mujer de 36 años, con antecedentes de tabaquismo leve, enfermedad celiaca, enfermedad de Graves que recibió tratamiento con drogas anti-tiroideas, posterior tiroidectomía total e hipotiroidismo y vasculitis ANCA C positiva (enfermedad de Wegener limitada). En tratamiento con meprednisona $60 \mathrm{mg} /$ día y ciclofosfamida $2 \mathrm{mg} / \mathrm{kg} /$ día 1 mes previo a su internación. Ingresó a UTI por disnea súbita y tos asociado a estridor laríngeo inspiratorio, sin sibilancias. Presentó 6 crisis de diez minutos en aproximadamente dos horas, permaneciendo asintomática intercrisis. En la fibrolaringoscopía hasta cuarto anillo traqueal no se observaron lesiones y la movilidad de las cuerdas impresionó normal fuera de la crisis. En la TAC de cuello se observó disminución del calibre en región glótica en inspiración en forma simétrica y la traquea era normal. La paciente no presentó clínica de RGE y este se diagnóstico mediante SEGD. Se realizó EFR que mostró obstrucción moderada fija, y no se observó alteración de la curva inspiratoria. Permanece en tratamiento con corticoides, beta 2 inhalados de larga duración y omeprazol 40 $\mathrm{mg}$ /día. No se realizó tratamiento psicológico ni fonoudiológico. Se encuentra asintomática desde hace 4 meses.

\section{DISCUSIÓN}

Morris y cols. (3) en una revisión extensa del tema, reunieron 1161 casos hasta el año 2006, reportando una predominancia femenina de 2:1. El 71\% fueron adultos, con una edad media de 34 años (rango entre 20-50 años), el resto fueron menores de 18 años, en su mayoría adolescentes. La verdadera incidencia de DCV en la población general no está definida, aunque se cree que el cuadro estaría subdiagnósticado

Maschka y cols. (4). clasificaron las causas de DCV en orgánicas (asma, ejercicio, irritantes [amonio, solventes, humo], RGE, infección de VAS, postoperatorio) y psicogénicas (desor- denes de conversión, estrés emocional, depresión, abuso físico, psicológico y sexual) (Tabla I). Nos detendremos en el asma por ser el diagnóstico diferencial más importante y en el RGE por ser la causa subyacente en nuestros pacientes.

\section{TABLA I}

CLASIFICACIÓN DE DISFUNCIÓN DE LAS CUERDAS VOCALES

-Causas orgánicas
Compresión de tronco (Ej: Arnold Chiari)
Lesión de neurona motora superior o cortical (Ej: Acci-
dente cerebrovascular)
Lesión de neurona motora inferior o nuclear (Ej: Distonía
laringea, ELA, MG, IM)
Desorden de los movimientos (DRLA, Parkinson Plus,
Blefarospasmo, etc)
Reflujo gastroesofágico
Otras (asma, ejercicio, irritantes, infección de VAS, pos-
toperatorio)
-Causas no orgánicas
Desordenes ficticios
Desordenes de conversión o somatización

ELA: esclerosis lateral amiatrófica; MG: miastenia gravis; IM: infarto medular; DRLA: dístonia respiratoria laríngea aductora; etc: ecetera; Ej: ejemplo.

En la serie de Newman y cols. (1) de 95 pacientes con DCV, se encontró que en el $56 \%$, ésta coexistía con asma (basándose en pruebas de broncoprovocaciòn y variabilidad del pico-flujo), y un $44 \%$ de los pacientes con DCV habían sido previamente diagnosticados como asmáticos con una demora diagnóstica de 5 años, el 28\% de estos requirieron intubación. En un estudio realizado en una sala de emergencias donde se evaluaron casos sospechosos de DCV se observó que un $20 \%$ de 50 pacientes presentaban evidencia laringoscópica de DCV (5). El 60\% de estos pacientes padecían DCV asociado a asma (basado en valores espirométricos). Típicamente la DCV se puede confundir con un asma inducido por ejercicio, inclusive con asma refractario a tratamiento con esteroides, lo que es muy frecuente (6). A diferencia del asma los pacientes con DCV no producen esputo, rara vez tienen síntomas nocturnos y las sibilancias se localizan en la parte superior del tórax (7). Los episodios de DCV tienen un comienzo abrupto y su resolución no presenta el típico empeoramiento durante las siguientes horas o días, característico de las crisis asmáticas $(1,7)$. Lo que es interesante es que el ejercicio así como la histamina y la metacolina pueden inducir tanto DCV como broncoconstricción lo que hablaría de una base fisiopatológica común (3).

En cuanto al reflujo gastroesofágico es importante recordar que los síntomas clásicos están ausentes en el 40-60\% de los pacientes con asma inducido por RGE, en el 57-94\% de los pacientes con manifestaciones de garganta, nariz y oído y en el 43-75\% de los pacientes con tos crónica por RGE (8). Se ha demostrado que solo el $25 \%$ de los pacientes con DCV secundaria a RGE tienen síntomas típicos de este último (9). La DCV inducida por RGE fue descripta en 1977 por Chodosh (10). En una serie retrospectiva de 39 pacientes con diagnóstico de DCV, el 92\% presentó RGE (3). En otra serie prospectiva se incluyeron 12 pacientes con DCV, todos fueron evaluados con un examen de phmetría, SEGD y laringos- 
copia (9). En la totalidad de los pacientes se hallaron signos típicos de RGE y en 10 la phmetria fue positiva. Diez de los 12 respondieron al tratamiento médico y sólo 2 requirieron funduplicatura de Nissen con la resolución completa de los síntomas. Más allá de los síntomas de laringoespasmo, solo un tercio de los mismos referían síntomas de RGE (9). Recientemente Suttithawil y cols. (11) confirmaron la relación causal del RGE al desencadenar un episodio agudo de DCV, utilizando el test de Bernstein. La frecuente asociación de la DCV con el RGE demuestra la importancia de considerar a este último como una potencial etiología de dichos episodios, realizar una prueba confirmatoria, e iniciar tratamiento con omeprazol dos veces al día.

Los síntomas más comunes son sibilancias, tos, disnea episódica, estridor inspiratorio, disfonía, y dolor torácico $(1,3)$. Se ha reportado la tos crónica como síntoma aislado (3). Estos síntomas son habitualmente interpretados como asma, y los pacientes son tratados durante varios años con esteroides con sus efectos adversos potenciales. Existen también síntomas secundarios a hiperventilación. Aunque la presentación suele ser dramática, habitualmente es autolimitada y no pone en riesgo la vida. A pesar del esfuerzo respiratorio de estos pacientes, solo rara vez se encuentran hipoxémicos o cianóticos. En los pacientes con déficit neurológico se debe descartar el compromiso del SNC como causa de DCV $(1,3)$.

Los diagnósticos diferenciales para estos síntomas se pueden localizar tanto en la vía aérea superior (orofaringe: angioedema, bocio; laringe: anafilaxia, parálisis de cuerdas bilateral, epiglotitis entre otros) como en la inferior (traquea: estenosis subglótica, traqueomalacia, cuerpos extraños; bronquios: asma, carcinoide entre otros) (3).

La patogénesis de la DCV no está bien determinada $(1,3)$. Los episodios de DCV pueden precipitarse por factores orgánicos (RGE, ejercicio, irritantes de VAS) como no orgánicos (desordenes psicológicos, estrés emocional), a través de diferentes procesos fisiopatológicos. La DCV se caracteriza por hiperreactividad laríngea de tipo colinérgico, lo que no explicaría por si sola el cuadro. Ayres y cols. (12) sugieren que un disbalance autonómico, con exceso de actividad en las regiones centrales (medula, tronco y el área 25 de la corteza precentral) estaría involucrado en el DCV. Por lo tanto, una combinación entre la hiperreactividad laríngea por un factor inflamatorio inicial asociado a un disbalance autonómico con una respuesta laríngea exagerada y repetitiva con el objetivo de proteger la vía aérea y al pulmón. Así, tanto algún factor inflamatorio como algún estimulo emocional estresante a nivel central desencadenaría episodios de DCV. Otra hipótesis plantea que el estimulo por irritantes de las terminales sensitivas nerviosas en el tracto de la vía aérea tanto superior como inferior, desencadenaría reflejos locales que producirían la obstrucción laríngea paroxística (3). Se sugiere que la estimulación repetida de este circuito llevaría a una alteración permanente a nivel genómico, produciendo un estado de perpetua hiperexcitabilidad en los centros de control neurológicos de la laringe, denominado como plasticidad neurológica que determina el síndrome de irritabilidad laríngea. En una serie retrospectiva se ha demostrado que un $25 \%$ de los pacientes presentaban alguna anormalidad estructural laríngea concurrente como estenosis subglótica, nódulos en cuerdas vocales, laringomalacia y laringitis posterior por RGE (13).

La visualización de las cuerdas vocales con un laringoscopio flexible durante un episodio de DCV es el patrón oro para confirmar el diagnóstico (1,3-5). La primera visualización directa en un paciente con DCV fue realizada por Mackenzie en 1869 (3). El hallazgo clásico es la aducción de los dos tercios anteriores de las cuerdas vocales, presentando algunas veces en el tercio posterior una forma de diamante (3). Los gases y la radiografía de tórax solo son de importancia en el diagnóstico diferencial de la disnea. La utilidad del EFR es limitado en el diagnóstico de DCV, dado la naturaleza paroxística del cuadro. La curva de flujo volumen inspiratoria (CFVI) $(1,3)$, es normal en el paciente asintomático. En el caso del paciente sintomático se observa disminución en el volumen espiratorio forzado del primer segundo (FEV1) y en la capacidad vital forzada $(\mathrm{CVF})$, conservándose el cociente FEV1/CVF. Es característico el hallazgo de una CFVI truncada, compatible con una obstrucción extra-torácica. En un paciente sintomático esto puede ser sugestivo del diagnóstico de DCV. A pesar de ello, en la revisión de Morris y cols. (3) sobre 1.161 pacientes, solo el $28,2 \%$ presentó estas alteraciones características en la CFVI. Newman, y cols. (1) reportaron un $23 \%$ de incidencia de alteración de la CFVI en pacientes asintomáticos. Estos hallazgos no han sido evaluados de forma prospectiva.

En cuanto al tratamiento hay que diferenciar el cuadro crónico del agudo. Se ha demostrado que el reaseguro es suficiente para paliar los síntomas obstructivos (3). Se coloca al paciente en posición de estornudo, pronunciando la letra "s". Otras medidas incluyen la oxigenoterapía, y las mezclas de Helio. También ha demostrado ser de utilidad la presión positiva intermitente y/o continua $(1,3)$. La utilización de sedantes como pequeñas dosis de propofol, lidocaina tópica y benzodiacepinas han demostrado ser efectivas para controlar episodios agudos de DCV. La experiencia con toxina botulínica tipo A es limitada, se ha reportado su uso en 9 pacientes (14). Esta toxina previene la liberación de acetilcolina en las terminales nerviosas, produciendo una denervación química del músculo tiroaritenoideo lo que impide la aducción de las cuerdas vocales. Es de utilidad en pacientes que requieren repetidas intubaciones e incluso traqueostomía, y que no responden al tratamiento convencional (14). Basados en la fisiopatología de la hiperreactividad laríngea se ha sugerido la utilización de anticolinérgicos como el ipratropio o tiotropio (12). Los corticoides no son de utilidad. En una revisión de casos reportados solo el 6,2\% (de 1.161 casos) requirió intubación, y el 3\% traqueotomía. Habitualmente son extubados en 24 horas. El manejo crónico de la DCV requiere de un enfoque multidiciplinario. Es importante educar al paciente para reconocer y prevenir el ataque agudo. Los ejes del tratamiento son las terapias fonoudiológicas y la psicoterapia. Se utilizan técnicas adaptadas del tratamiento de los trastornos funcionales de la voz. En un estudio prospectivo con 20 pacientes se utilizó terapia fonoaudiológica y terapia familiar, el 95\% controlaron los síntomas y el $65 \%$ discontinuo el uso de medicación para el asma (15). La psicoterapia forma parte del tratamiento de primera línea para la DCV. La efectividad de la psicoterapia para disminuir o eliminar los episodios de DCV no ha sido evaluada en forma prospectiva. Otros tratamientos descriptos son el biofeedback, las técnicas de relajación, y la hipnosis que podrían utilizarse como complemento de la psicoterapia (3).

No existen series prospectivas que mencionen el pronóstico a largo plazo. Algunos pacientes presentan síntomas autolimitados y responden rápidamente a la terapia fonoaudiológica y psicológica. Muchos pacientes no responden al 
tratamiento convencional y continúan con síntomas recurrentes. No se ha determinado si existen diferencias teniendo en cuenta la causa (orgánica vs no orgánica) subyacente de la DCV, en la resolución de los síntomas o en el pronóstico a largo plazo. Es pasible de pensar que si existe un desencadenante orgánico tratable, el cuadro pueda ser controlado y evolucione favorablemente. La DCV es una causa importante de síntomas respiratorios agudos y crónicos, y debe ser considerada entre los diagnósticos diferenciales de disnea. Reconocer esta entidad como diagnóstico diferencial es especialmente importante en el caso de pacientes asmáticos que no responden al tratamiento estándar. La DCV fue clásicamente considerada un trastorno psicológico. Nueva evidencia muestra diferentes mecanismos orgánicos que explicarían dicha disfunción y que deben ser evaluados ya que son pasibles de tratamiento específico.

\section{Bibliografía}

1. Newman KB, Mason UG, Schmaling KB. Clinical features of vocal cord dysfunction. Am J Respir Crit Care Med 1995; 152: 1382-6.

2. Dunglison RD. The Practice of Medicine. In: Lea \& Blanchard. Philadelphia: 1842. p. 257-8.

3. Morris MJ, Allan PF, Perkins PJ. Vocal Cord Dysfunction. Etiologies and Treatment. Clin Pulm Med 2006; 13: 73-86.

4. Maschka DA, Bauman NM, McCray PB, et al. A Classification Scheme for Paradoxical Vocal Cord Motion. Laryngoscope 1997; 107: 1429-35.

5. Jain S, Bandi V, Officer T, et al. Incidence of vocal cord dysfunction in patients presenting to emergency room with acute asthma exacerbation. Chest 1999; 116: 243-4.

6. Morris MJ, Deal LE, Bean DR, et al. Vocal Cord Dysfunction in patients with exertional dyspnea. Chest 1999; 116: 1676-82.

7. Reisner C, Nelson HS. Vocal cord dysfunction with nocturnal awakening. J Allergy Clin Immunol 1997; 99: 3-6.

8. Young P, Finn BC, Bruetman JE, Trimarchi H. Gastroesophageal reflux as a cause of night sweting. An Med Interna 2007; 24: 285-8.

9. Loughlin CJ, Koufman JA. Paroxismal Laryngospasm Secondary to
Gastroesophageal Reflux. Laryngoscope 1996; 106: 1502-5.

10. Chodosh PL. Gastro-esophage-pharingeal reflux. Laryngoscope 1977; 87: $1418-27$

11. Suttithawil W, Chakkaphak S, Jaruchinda P, Fuangtong R. Vocal cord dysfunction concurrent with a nutcracker esophagus and the role of gastroesophageal reflux disease. Ann Allergy Asthma Immunol 2006; 96: 373-5.

12. Ayres JG, Gabbott PLA. Vocal cord dysfunction and laryngeal hyperresponsiveness: a function of altered autonomic balance? Thorax 2002; 57: 284-5.

13. Patel NJ, Jorgensen C, Kuhn J, et al. Concurrent laryngeal abnormalities in patients with paradoxical vocal fold dysfunction. Otolaryngol Head Neck Surg 2004; 130: 686-89.

14. Maillard I, Schweizer V, Broccard A, et al. Use of botulinum toxin type A to avoid tracheal intubation or tracheostomy in severe paradoxical vocal cord movement. Chest 2000; 118: 874-6.

15. Sullivan MD, Heywood BM, Beukelman DR. A treatment for vocal cord dysfunction in female athletes: an outcome study. Laryngoscope 2001; 111: 1751-5. 